\title{
Impact on Medical Cost, Cumulative Survival, and Cost-Effectiveness of Adding Rituximab to First-Line Chemotherapy for Follicular Lymphoma in Elderly Patients: An Observational Cohort Study Based on SEER-Medicare
}

\author{
Robert I. Griffiths, ${ }^{1,2}$ Michelle L. Gleeson, ${ }^{1}$ Joseph Mikhael, ${ }^{3}$ and Mark D. Danese ${ }^{1}$ \\ ${ }^{1}$ Outcomes Insights Inc., 340 N. Westlake Boulevard, Suite 200, Westlake Village, CA 91362, USA \\ ${ }^{2}$ Division of General Internal Medicine, Johns Hopkins University School of Medicine, 733 North Broadway, Baltimore, \\ MD 21205, USA \\ ${ }^{3}$ Mayo Clinic in Arizona, 13400 E. Shea Bloulevard, 3rd Floor, Scottsdale, AZ 85259, USA
}

Correspondence should be addressed to Robert I. Griffiths, bob@outins.com

Received 30 April 2012; Accepted 11 July 2012

Academic Editor: P. Vineis

Copyright (c) 2012 Robert I. Griffiths et al. This is an open access article distributed under the Creative Commons Attribution License, which permits unrestricted use, distribution, and reproduction in any medium, provided the original work is properly cited.

Rituximab improves survival in follicular lymphoma (FL), but is considerably more expensive than conventional chemotherapy. We estimated the total direct medical costs, cumulative survival, and cost-effectiveness of adding rituximab to first-line chemotherapy for FL, based on a single source of data representing routine practice in the elderly. Using surveillance, epidemiology, and end results (SEER) registry data plus Medicare claims, we identified 1,117 FL patients who received first-line CHOP (cyclophosphamide (C), doxorubicin, vincristine (V), and prednisone (P)) or CVP +/- rituximab. Multivariate regression was used to estimate adjusted cumulative cost and survival differences between the two groups over four years after beginning treatment. The median age was 73 years (minimum 66 years), 56\% had stage III-IV disease, and 67\% received rituximab. Adding rituximab to first-line chemotherapy was associated with higher adjusted incremental total cost $(\$ 18,695 ; 95 \%$ Confidence Interval (CI) $\$ 9,302-\$ 28,643)$ and longer adjusted cumulative survival (0.18 years; 95\% CI 0.10-0.27) over four years of followup. The expected cost-effectiveness was \$102,142 (95\% CI \$34,531-296,337) per life-year gained. In routine clinical practice, adding rituximab to first-line chemotherapy for elderly patients with FL results in higher direct medical costs to Medicare and longer cumulative survival after four years.

\section{Introduction}

Like many countries, the United States is struggling with the rising costs of cancer care [1]. Nowhere is this more apparent than within Medicare, the primary payer of health services for those of age $\geq 65$ years, where the rise in spending on cancer drugs over the past 15 years has been singled out as a leading cause of increased spending overall [2]. Presently, Medicare's ability to control rising spending on cancer drugs is limited [2], and it is also well known that Medicare does not formally consider cost or cost-effectiveness as a criterion for coverage and reimbursement [1]. Since most new drugs cost considerably more than older drugs $[2,3]$, and since many are added to rather than substituted for drugs in existing regimens, the net cost impact of new drugs will be determined primarily by the cost of the drug itself, and secondarily by the extent to which those costs are augmented or offset by its clinical benefits. In cancer care, clinical benefits that have cost implications include reducing the rate of disease progression or relapse after first-line therapy and improving survival.

It is standard practice to include all of these factors in cost-effectiveness analyses of new cancer drugs. However, such analyses often are conducted early in the commercial 
life-cycle of the drug, and in the absence of comprehensive data from routine clinical practice, usually they are based on models that incorporate data from multiple sources, including drug regimens and clinical outcomes from phase III trials. While these analyses provide important insight into the potential cost and cost-effectiveness of new drugs in routine clinical practice, and may have a high level of internal validity due to the sources of clinical data used, invariably they will lack some external validity. This is due to differences in the types of patients treated, for example, patient age, cancer stage, the approaches to managing disease progression or relapse, and the net cost impact of improving overall survival in patients with higher comorbidity burdens.

In 1997, rituximab became the first therapeutic antibody approved for the treatment of cancer [4]. Initially, it was approved for treating relapsed or refractory, CD20+, B-cell low grade, non-Hodgkin's lymphoma (NHL). Since then, the indication has expanded several times to include firstline therapy, follicular lymphoma (FL), chronic lymphocytic leukemia (CLL), and rheumatoid arthritis [4]. The efficacy of rituximab is well-supported by data from randomized clinical trials. For instance, addition of rituximab to first-line chemotherapy has been shown in clinical trials of DLBCL [58 ] to improve progression-free and overall survival in elderly patients, where reported risk-ratios for all-cause mortality range from 0.53 (95\% confidence interval (CI) 0.37-0.77) [5] to $0.72(95 \%$ CI $0.52-1.00)$ [6], and in younger patients [7]. The efficacy of rituximab as first-line therapy for advanced FL was established in several randomized trials [9-12]. The German Low Grade Lymphoma Study Group compared CHOP (cyclophosphamide (C), doxorubicin, vincristine $(\mathrm{V})$, and prednisone $(\mathrm{P}))(n=205)$ to R-CHOP $(n=223)$ in a cohort of patients with untreated, advanced-Stage III/IV FL [9]. They showed a statistically significant improvement in overall survival, with 6 deaths in the R-CHOP group compared to 17 deaths in the CHOP group within the first three years. Another phase III study compared CVP alone $(n=162)$ to R-CVP $(n=159)$ in a similar cohort of untreated FL patients [11]. In that study, the percent of patients; surviving four years was significantly higher in the R-CVP (83\%) versus the CVP-alone group (77\%) $(P=$ $0.029)$.

The effectiveness of rituximab in routine clinical practice has been evaluated in several studies using the National Cancer Institute's (NCI) surveillance, epidemiology, and end results (SEER) Medicare database [13-15]. They indicate that the survival benefits observed in clinical trials extend to elderly patients treated in routine clinical practice, and to mantle cell lymphoma (MCL) [15] where survival benefits have not been demonstrated in clinical trials. They also show that even though rituximab was not approved initially for first-line treatment, its use as part of first-line chemotherapy increased rapidly after Medicare began coverage in 1998, and now the majority of SEER-Medicare patients who receive chemotherapy as first-line therapy for FL, MCL, and CLL also receive rituximab [13-15].

Efficacy and effectiveness notwithstanding, rituximab is an expensive addition to first-line therapy for NHL with an estimate of $\$ 24,034$ in FL [16] and \$13,900 in DLBCL [17] based on the projections of cost-effectiveness models, and in part dependent on the year of the analysis and number of administrations of rituximab. The current Medicare allowed amount (including 20\% patient copayment) for rituximab is $\$ 611$ per $100 \mathrm{mg}$ vial [18]. At a recommended dose of $375 \mathrm{mg} / \mathrm{m}^{2}$ body surface area (BSA) [19], and assuming an average BSA of $1.72 \mathrm{~m}^{2}$ [16] and 6-8 cycles per first-line course of rituximab [20], the current cost to Medicare could be $\$ 20,530-\$ 27,373$ per course, an estimate that excludes the $20 \%$ copayment. A disproportionate number of patients diagnosed with NHL are aged $\geq 65$ years, including $50 \%$ of the estimated 129,500 prevalent and $59 \%$ of the 21,800 incident DLBCL patients and 52\% of FL patients [21, 22]. Consequently, Medicare is an important payer for therapy and other cancer care in NHL, and rituximab is an important line item for Medicare. In 2009, rituximab accounted for the largest percent of Medicare part B expenditures (7.8\%) of any drug administered in physicians' offices or furnished by suppliers [23].

Given the efficacy and cost of rituximab, there has been considerable interest in evaluating its net cost impact and cost-effectiveness, and studies have been conducted in DLBCL [17, 24-27] and FL [16, 28, 29]. Several studies of adding first-line rituximab to chemotherapy in DLBCL projected cost offsets [17, 24-27], and in one instance savings [26], associated with lower rates of progression/relapse and improved survival. Two cost-effectiveness analyses of rituximab in combination with chemotherapy have been conducted in FL, one in the US, and one in the United Kingdom $[16,28]$. Both were based on computer models, and incorporated data from a number of sources including clinical data from randomized trials. In the US, Hornberger and colleagues projected adding rituximab to CVP would result in a lifetime cost difference of $+\$ 26,439$, the majority of which was due to rituximab itself $(\$ 24,034)$, a difference in mean overall survival of +1.51 years, and a cost per qualityadjusted life-year gained of $\$ 28,565$ [16].

The objective of the present study was to estimate the cost and cost-effectiveness of rituximab added to firstline chemotherapy for FL, using a single source of data representative of routine clinical practice in elderly patients in the US. It differs from previous economic studies of rituximab in NHL, the majority of which were based on models, incorporated data from multiple sources including clinical trials, and projected cost and survival beyond the available data.

\section{Materials and Methods}

2.1. Data Source. The source of data for this study was the National Cancer Institute's (NCI) surveillance, epidemiology, and end results (SEER) cancer registry linked to Medicare administrative and claims data [30]. Presently, SEER contains cancer incidence and survival data from 17 population-based cancer registries throughout the United States covering approximately $28 \%$ of the population [31]. The registries routinely collect data on patient demographics, 
primary tumor site, tumor morphology and stage at diagnosis, first course of treatment, and followup for vital status. In SEER-Medicare, cancer registry data are linked to Medicare enrollment and claims data, which are available for $93 \%$ of those aged $\geq 65$ years in the SEER registry [32]. At the time this study was performed, the SEER-Medicare linkage included all Medicare-eligible persons appearing in the SEER data through 2005 and their Medicare claims through 2007.

Medicare claims files linked to SEER consist of the following: medicare provider analysis and review (MEDPAR) all hospital (part A) short stay, long stay, and skilled nursing facility bills; national claims history $(\mathrm{NCH})$-all physician/supplier (part B) bills; Outpatient, which includes all bills from institutional outpatient (part B) providers; home health agency (HHA) - all claims for home health services; hospice; and durable medical equipment (DME). The Medicare benefit for oral drugs (part D) began on January 1, 2006, and claims for oral drugs without an intravenous equivalent were not available for our study.

2.2. Inclusion Criteria. Patients were included if they were diagnosed with FL [33] between January 1, 1999, and December 31, 2005, FL was the first primary cancer diagnosed, they began infused CHOP (cyclophosphamide $(\mathrm{C})$, doxorubicin, vincristine $(\mathrm{V})$, and prednisone $(\mathrm{P}))$ or CVP chemotherapy with or without rituximab within 90 days following diagnosis, and they survived at least 60 days after first claim indicating the beginning of therapy. We required a minimum 60 days survival after the first claim to classify the initial treatment regimen. In addition, to ensure complete claims history for purposes of calculating an NCI comorbidity index score $[34,35]$, patients had to have been enrolled in both Medicare parts A and B, with no health maintenance organization (HMO) coverage, for 12 months prior to FL diagnosis.

To preserve patient confidentiality, SEER reports only the calendar month of diagnosis. Therefore, we set the date of diagnosis to the first day of that month. Identification of FL was made using the World Health Organization (WHO) International Classification of Diseases for Oncology, 3rd Edition (ICD-O-3) histology codes 9695 (FL Grade 1), 9691 (FL Grade 2), 9698 (FL Grade 3), and 9690 (FL not otherwise specified) [33].

2.3. Observation Period. Patients were followed for up to four years from the day first-line therapy began, or until death, the end of their claims (December 31, 2007), or the end of their Medicare part A and/or part B coverage, whichever came first. The date of death was assigned by using the Medicare date because it is considered more current than the SEER date.

2.4. Patients and Variables. Patients were described according to their demographic, clinical, and socioeconomic characteristics. Requiring eligible patients to have at least one year of Medicare enrollment prior to diagnosis meant that the minimum age in the cohort was 66 years. Patients were classified by stage and extranodal involvement (yes/no) at
FL diagnosis. In the absence of hemoglobin level, serum lactate dehydrogenase (LDH) level, or the number of nodal areas, three of the five prognostic factors for mortality in the follicular lymphoma international prognostic index (FLIPI) [36], we used Medicare claims to identify several predictors of poor performance status, including the use of oxygen and related respiratory therapy supplies, wheel chair and supplies, home health agency, and skilled nursing facility, all from 12 months before until 30 days after FL diagnosis [37]. Individual services were combined into a score of 0 (none) or 1 (use of any service). Medicare claims also were used to identify anemia [14], and to calculate an NCI comorbidity index score $[34,35]$ for each patient. SEER-Medicare contains information from the 2000 Census, reported at the tract level in which the patient lives, for the percent of the population living in poverty and the percent of those aged 25 years or older with some college. We used these as indicators of the socioeconomic status of individual patients in the cohort. The assigned metropolitan statistical area served as a geographic indicator.

2.5. First-Line Therapy. We searched the Medicare outpatient and $\mathrm{NCH}$ files to identify claims containing healthcare common procedure coding system (HCPCS) "J" codes for cyclophosphamide (J8530, J9070, J9080, J9090-J9097), doxorubicin (J9000, J9001), and vincristine (J9370, J9375, J9380). All such claims within 60 days after the first were used to classify the patient's first-line regimen as rituximab plus chemotherapy (CHOP or CVP) or chemotherapy alone. In the absence of data on oral therapy, prednisone was assumed to have been included in the regimen when the other three chemotherapy agents were present.

2.6. Cost. The total direct medical cost to Medicare, from the beginning of first-line therapy until the end of the observation period (maximum four years), was estimated based on Medicare paid amounts obtained from bills within the claims files. Diagnosis and procedure codes within claims were used to identify three mutually exclusive categories of costs: immunochemotherapy, which included the costs of infused cancer therapy agents provided during firstline and any subsequent treatment, other cancer care (any claim with an International Classification of Diseases, 9th Revision, Clinical Modification [ICD-9-CM] diagnosis code 140.xx-208.xx [malignant neoplasms], but no code for immunochemotherapy), and noncancer care (any other claim). All paid amounts were inflated to 2009 US dollars based on the hospital input price index for part A claims and the medical expenditure index for part B claims [38]. Since SEER-Medicare data are limited in time, our approach to estimating population mean costs accounted for censoring in the data using inverse probability weighting (IPW) [3945].

2.7. Survival. Conventional approaches to analyzing adjusted differences in survival between alternative treatments are based primarily on proportional hazards regression. However, this approach does not produce an estimate of the 
adjusted difference in overall survival between two treatment groups at specific time-points, as required for performing cost-effectiveness analysis directly from observational data. In this study, we used inverse probability of treatment weighting (IPTW) for survival data [46] to estimate the adjusted difference in cumulative survival between the two treatment groups. First, we estimated the conditional probability of assignment to the rituximab plus chemotherapy group for each patient using logistic regression with treatment group as the dependent variable and all other independent variables from the cost model as predictor variables. Then, the inverse of this conditional probability estimate was assigned as a weight to each patient, and it was used to adjust the Kaplan-Meier cumulative survival estimate for each treatment group.

2.8. Statistical Analysis. Chi-square analysis was used to test for the independence between patient characteristics specified as categorical variables and the two first-line treatment groups. Analysis of variance was used to test for independence of continuous variables.

We performed partitioned IPW least squares regression analysis to examine adjusted associations between cumulative costs over four years and patient demographic, clinical, and treatment factors. Separate regression analysis was performed for each of the 48 monthly partitions. Coefficients for each patient factor were summed across the 48 partitions to obtain the cumulative, incremental cost associated with that factor. Confidence intervals (CI) for the cumulative cost coefficients were calculated by a bootstrap approach [47], in which the process of performing 48 partitioned regression analyses and summing coefficients across partitions was repeated 1,000 times using sampling with replacement from the original cohort. This process was applied to total costs and each of the three cost categories, for a total of 192,000 regressions.

Using the adjusted Kaplan-Meier estimator based on IPTW, we estimated the adjusted difference in survival between rituximab plus chemotherapy and chemotherapy alone in each of 48 monthly partitions following the beginning of first-line therapy. Since patients in this study were required to have survived at least 60 days after the beginning of first-line therapy, by definition the difference in survival was zero during the first two months. The cumulative survival difference was calculated by summing the adjusted survival differences. We obtained 95\% CIs for the partitioned incremental survival estimates from the weighted log rank statistic [46].

Cost effectiveness ratios were calculated in months 3 48 (ignoring months 1-2 due to the inclusion criteria; there was no mortality during this period) after the beginning of first-line therapy, by dividing the incremental cumulative cost of rituximab by the cumulative survival difference. Confidence intervals for the cost-effectiveness ratio were calculated by dividing the upper bound of the $95 \%$ CI for cost by the lower bound of the $95 \%$ CI for survival, and then repeating the process with the lower cost and upper survival bounds.

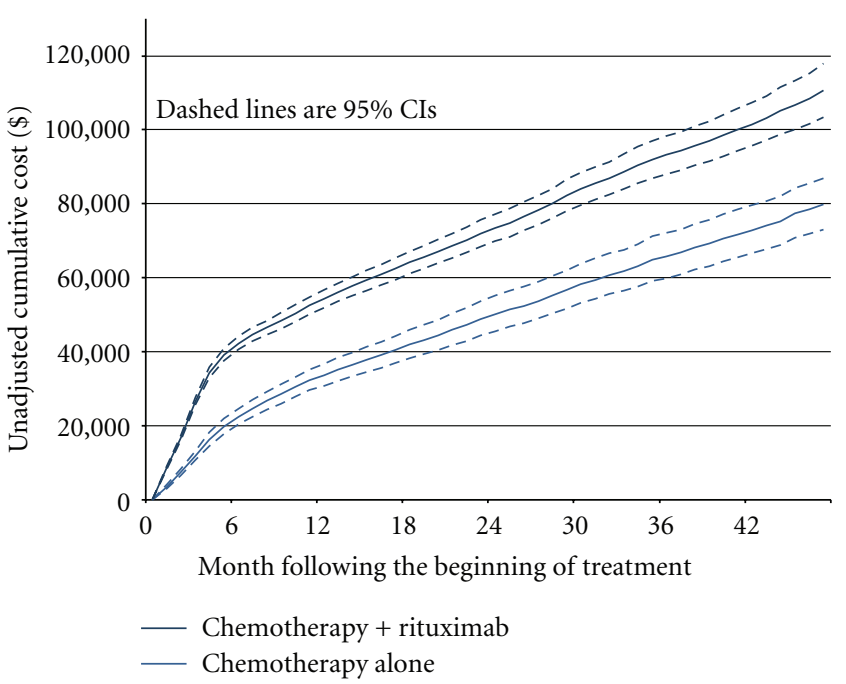

Figure 1: Unadjusted cumulative cost.

\section{Results}

3.1. Patients. There were 1,117 patients in the final cohort: $750(67 \%)$ received rituximab plus chemotherapy, and 367 (33\%) received chemotherapy alone (Table 1). The median age at diagnosis was 73 years (minimum 66 years), 56\% had Stage III/IV disease, $42 \%$ had extranodal involvement, and $15 \%$ had $\geq 1$ indicator of poor performance status. Rituximab patients were diagnosed later in the study period, and were more likely to have higher-grade histology. There were no differences in stage at diagnosis, extranodal involvement, or NCI Comorbidity Index score between the two groups.

3.2. Treatment. Overall, 67\% (754) received CHOP, and the remaining 33\% (363) received CVP. Rituximab patients were more likely to receive $\mathrm{CHOP}$ ( $72 \%$ versus $58 \%$ for chemotherapy alone; $P<0.0001$ ). Among those who received rituximab, the average number of rituximab administrations during first-line therapy was 6.3 (median 6; interquartile range [IQR] 5-7), and the average number of units was $7.3 \times 100 \mathrm{mg}$ vials (median 7; IQR 6-8).

3.3. Costs. The mean unadjusted IPW cumulative cost was $\$ 111,815$ (95\% CI $\$ 104,455-\$ 119,466)$ in the rituximab group compared to $\$ 80,826$ (95\% CI $\$ 74,006-\$ 88,113$ ) for chemotherapy alone (Figure 1). The mean cost of firstline rituximab was $\$ 15,640$ (median $\$ 14,786$; IQR $\$ 10,831-$ $\$ 18,560$ ), and the average cost per administration was $\$ 2,836$ (median \$2,747; IQR \$2,617-\$3,113).

In the multivariate analyses, rituximab was associated with statistically significantly higher incremental total $(\$ 18,695 ; 95 \%$ confidence interval [CI] \$9,302-\$28,643) and immunochemotherapy $(\$ 13,336 ; 95 \%$ CI $\$ 9,364-\$ 17,552)$ costs after four years, but not other cancer $(\$ 4,816 ; 95 \%$ CI $\$ 1,899-\$ 11,303)$ or noncancer $(\$ 351$; $95 \%$ CI $\$ 3,481-$ $\$ 4,202$ ) costs (Table 2). Most of the separation in total and immunochemotherapy costs between the two treatment groups occurred during the first 6 months after the beginning of first-line therapy (Figure 2). Thereafter, there was 


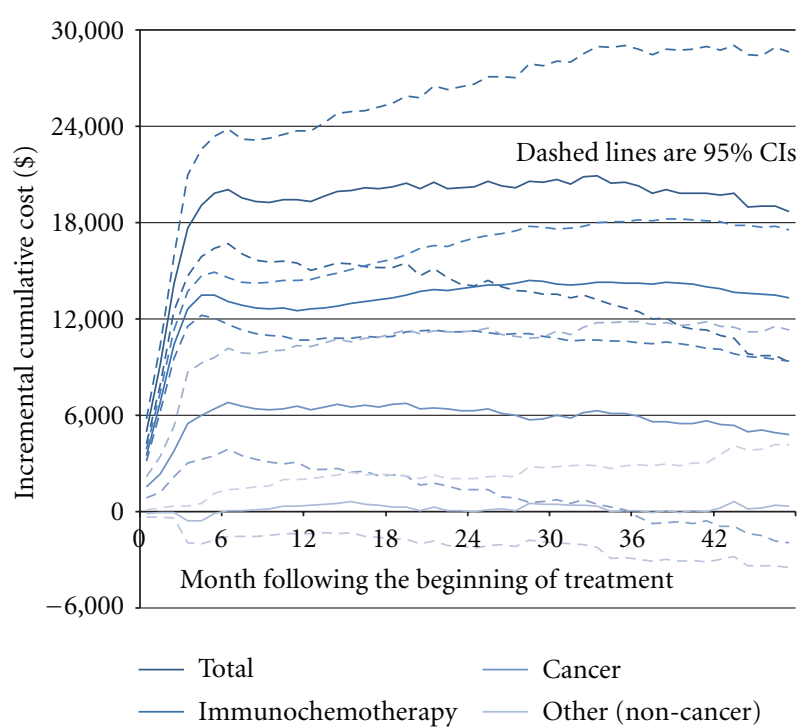

FIGURE 2: Incremental cumulative cost of adding rituximab to chemotherapy.

very little separation in total or immunochemotherapy costs. During the first 36 months, incremental costs of other cancer services were statistically significantly higher in the rituximab group. However, costs of other cancer services declined in later months, and by month 48 the difference between groups was not significant. There was no difference in non-cancer costs at any time during the observation period.

Other factors associated with higher costs were stage (immunochemotherapy cost), extranodal involvement (total and other cancer costs), NCI comorbidity index (predominately non-cancer cost), and $\geq 1$ indicator of poor performance status (total and other cancer costs). Factors associated with lower costs were age $>80$ years (immunochemotherapy cost), other race/ethnicity (total and immunochemotherapy costs), and urban/rural (compared to metropolitan) area (total and other cancer cost). Results of the multivariate analysis of total cost that included only patients treated with $\mathrm{CHOP}$ were similar to those that included all patients (Table 3).

3.4. Survival. Overall, unadjusted (crude) cumulative survival was $83 \%$ at 24 months and $73 \%$ at 48 months; $85 \%$ and $77 \%$, respectively, for rituximab plus chemotherapy; $79 \%$ and $66 \%$, respectively, for chemotherapy alone. The incremental cumulative adjusted survival (years) in the rituximab group was 0.05 (95\% CI 0.02-0.09) after two years, 0.11 (95\% CI 0.05-0.16) after three years, and 0.18 (95\% CI $0.10-0.27$ ) after four years of observation (Figure 3 ).

3.5. Cost per Life Year Gained. The cost per life year gained in the rituximab group was $\$ 382,642$ (95\% CI $\$ 164,900$ $\$ 1,360,559)$ after two years, $\$ 193,859$ (95\% CI $\$ 77,314-$ $\$ 609,607)$ after three years, and \$102,142 (95\% CI \$34,531$\$ 296,337$ ) after four years of observation (Figure 4). At no time point during the observation period did the $95 \%$ CI

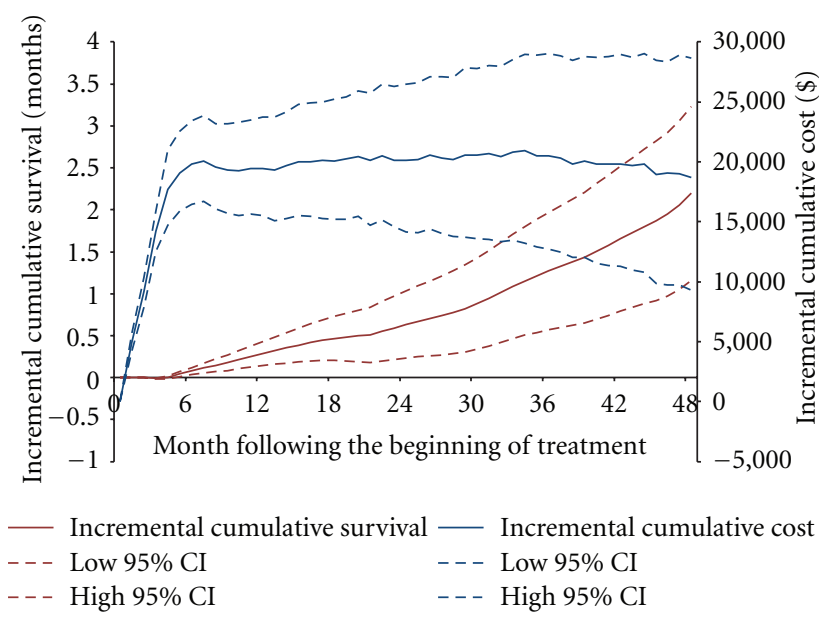

FIGURE 3: Incremental cumulative survival and total cost of adding rituximab to chemotherapy.

for the cost-effectiveness ratio contain a value indicating that rituximab plus chemotherapy was both more costly and less effective (dominated), or less costly and more effective (dominates) than chemotherapy alone.

\section{Discussion}

In this study, we identified a cohort of elderly FL patients who received first-line chemotherapy with or without rituximab. We followed this cohort for up to four years after the beginning of therapy to estimate the net cost impact and cost-effectiveness of adding rituximab to first-line therapy. Our findings indicate that over this time horizon, the initial cost of rituximab accounted for the majority of the cost difference between the two treatment groups. The mean cost of first-line rituximab in our study $(\$ 15,640)$ was substantially lower than expected based on the projections from a cost-effectiveness model in FL $(\$ 24,034)$ [16], and our own calculations based on recommended dosing and current Medicare reimbursement. Patients in our study received an average of 6.3 administrations and $7.3100 \mathrm{mg}$ vials per administration during first-line therapy. Hornberger and colleagues [16] assumed 8 administrations, $700 \mathrm{mg}$ per administration, and $\$ 5.82 / \mathrm{mg}$ in estimating the cost of firstline rituximab. Part of the difference may be explained by the fact that patients in our study received almost $25 \%$ fewer administrations. Another reason for the difference is that our calculations were made using Medicare paid amounts, which exclude the $20 \%$ patient copayment. Finally, although we inflated our costs to US\$2009, it is possible that our inflation factors did not adequately compensate for inflation in the price of rituximab during the study period. Dividing the mean cost of rituximab by the average number of milligrams delivered during the course of therapy $(4,599)$, the average cost per milligram was only $\$ 3.40$ in our study.

It is noteworthy that in the rituximab group the cumulative incremental cost of immunochemotherapy during the 
TABLe 1: Patient characteristics.

\begin{tabular}{|c|c|c|c|c|c|c|c|}
\hline & \multicolumn{6}{|c|}{ First-line therapy } & \multirow{3}{*}{$P$ value } \\
\hline & \multicolumn{2}{|c|}{ Overall $(N=1,117)$} & \multicolumn{2}{|c|}{$\begin{array}{l}\text { Rituximab plus chemotherapy } \\
\qquad(n=750)\end{array}$} & \multicolumn{2}{|c|}{$\begin{array}{l}\text { Chemotherapy alone } \\
\qquad(n=367)\end{array}$} & \\
\hline & $n$ & $\%(\mathrm{SD})$ & $n$ & $\%(\mathrm{SD})$ & $n$ & $\%(\mathrm{SD})$ & \\
\hline \multicolumn{8}{|l|}{ Age at diagnosis (years) } \\
\hline $66-70$ & 327 & 29.3 & 223 & 29.7 & 104 & 28.3 & \multirow{4}{*}{0.94} \\
\hline $71-75$ & 293 & 26.2 & 197 & 26.3 & 96 & 26.2 & \\
\hline $76-80$ & 285 & 25.5 & 191 & 25.5 & 94 & 25.6 & \\
\hline$>80$ & 212 & 19.0 & 139 & 18.5 & 73 & 19.9 & \\
\hline \multicolumn{8}{|l|}{ Gender } \\
\hline Male & 517 & 46.3 & 355 & 47.3 & 162 & 44.1 & \multirow{2}{*}{0.32} \\
\hline Female & 600 & 53.7 & 395 & 52.7 & 205 & 55.9 & \\
\hline \multicolumn{8}{|l|}{ Race/ethnicity } \\
\hline White & 1000 & 89.5 & 675 & 90.0 & 325 & 88.6 & \multirow{2}{*}{0.46} \\
\hline Nonwhite & 117 & 10.5 & 75 & 10.0 & 42 & 11.4 & \\
\hline \multicolumn{8}{|l|}{ Year of diagnosis } \\
\hline 1999-2002 & 540 & 48.0 & 231 & 30.8 & 309 & 84.2 & \multirow{2}{*}{$<0.0001$} \\
\hline $2003-2005$ & 577 & 51.7 & 519 & 69.2 & 58 & 15.8 & \\
\hline \multicolumn{8}{|l|}{ Stage at FL diagnosis } \\
\hline I & 236 & 21.1 & 160 & 21.3 & 76 & 20.7 & \multirow{5}{*}{0.42} \\
\hline II & 189 & 16.9 & 119 & 15.9 & 70 & 19.1 & \\
\hline III & 271 & 24.3 & 188 & 25.1 & 83 & 22.6 & \\
\hline IV & 350 & 31.3 & 240 & 32.0 & 110 & 30.0 & \\
\hline Unknown & 71 & 6.4 & 43 & 5.7 & 28 & 7.6 & \\
\hline \multicolumn{8}{|l|}{ Histology (grade) } \\
\hline 1 & 188 & 16.8 & 112 & 14.9 & 76 & 20.7 & \multirow{4}{*}{0.02} \\
\hline 2 & 274 & 24.5 & 175 & 23.3 & 99 & 27.0 & \\
\hline 3 & 345 & 30.9 & 248 & 33.1 & 97 & 26.4 & \\
\hline Not specified & 310 & 27.8 & 215 & 28.7 & 95 & 25.9 & \\
\hline \multicolumn{8}{|l|}{ Extranodal involvement } \\
\hline No & 573 & 51.3 & 382 & 50.9 & 191 & 52.0 & \multirow{3}{*}{0.37} \\
\hline Yes & 473 & 42.3 & 325 & 43.3 & 148 & 40.3 & \\
\hline Unknown & 71 & 6.4 & 43 & 5.7 & 28 & 7.6 & \\
\hline \multicolumn{8}{|c|}{ Presence of "B" symptoms } \\
\hline No & 477 & 42.7 & 348 & 46.4 & 129 & 35.2 & \multirow{3}{*}{$<0.001$} \\
\hline Yes & 185 & 16.6 & 129 & 17.2 & 56 & 15.3 & \\
\hline Unknown/Missing & 455 & 40.8 & 273 & 36.4 & 182 & 49.6 & \\
\hline \multicolumn{8}{|l|}{ Anemia } \\
\hline No & 1073 & 96.1 & 719 & 95.8 & 354 & 96.5 & 0.63 \\
\hline Yes & 44 & 3.9 & 31 & 4.1 & 13 & 3.5 & \\
\hline NCI comorbidity indes & & & & & & & \\
\hline 0 & 796 & 71.3 & 546 & 72.8 & 250 & 68.1 & \\
\hline 1 & 234 & 20.9 & 146 & 19.5 & 88 & 24.0 & 0.34 \\
\hline$\geq 2$ & 87 & 7.8 & 58 & 7.7 & 29 & 7.9 & \\
\hline Performance status ind & & & & & & & \\
\hline 0 & 955 & 85.5 & 648 & 86.4 & 307 & 86.7 & 0.22 \\
\hline$\geq 1$ & 162 & 14.5 & 102 & 13.6 & 60 & 32.9 & \\
\hline
\end{tabular}


TABle 1: Continued.

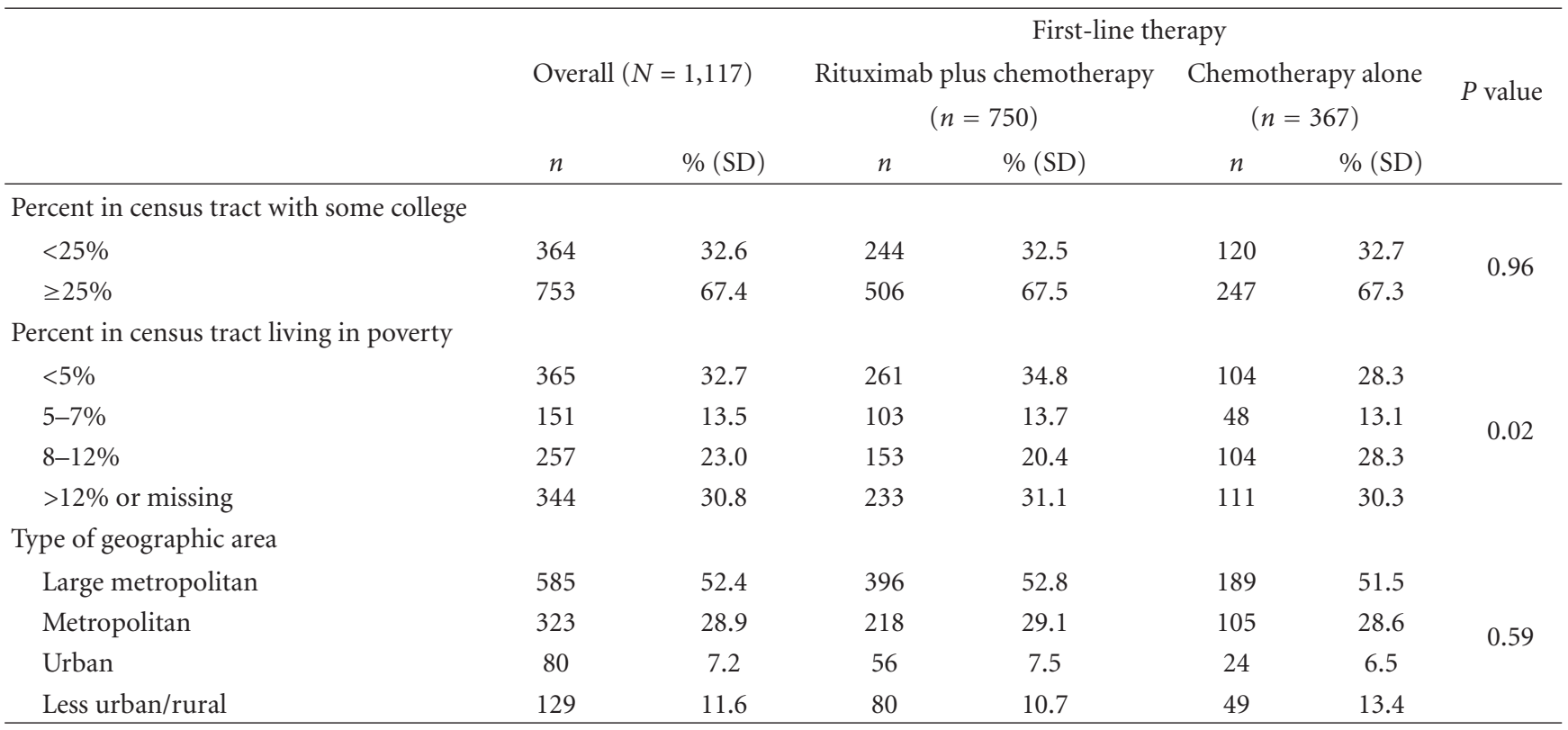

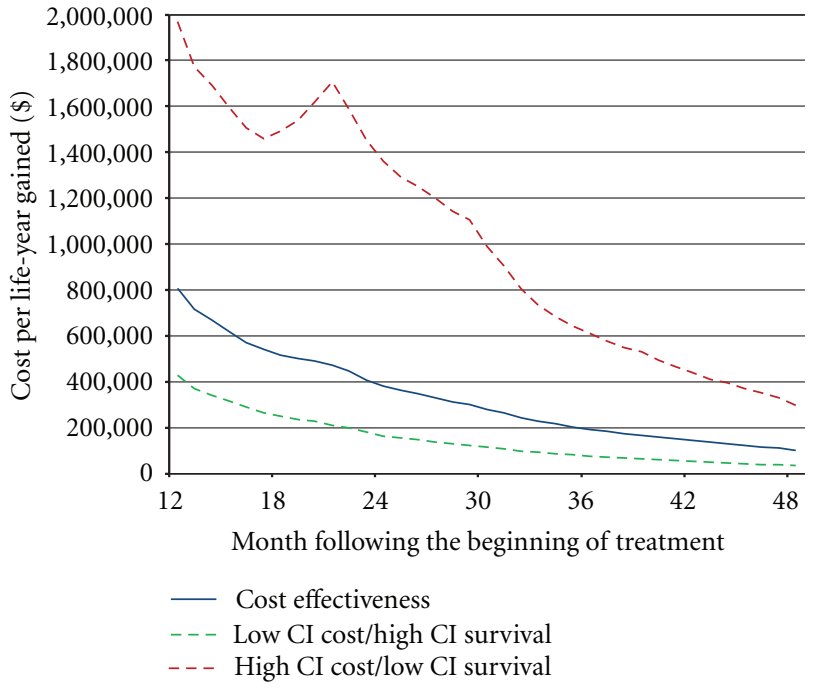

FIgure 4: Cost per life-year gained associated with adding rituximab to chemotherapy.

four-year period $(\$ 13,336)$ was lower than the cost of firstline rituximab $(\$ 15,640)$. This suggests the initial costs of rituximab were offset to some extent by higher treatment costs in the chemotherapy group due to higher rates of nonremitting and/or relapsing disease. The cumulative incremental cost of other cancer care also was significantly higher in the rituximab group through much of the study period. Most of the difference between the two groups appeared during first-line therapy, which suggests there may have been differences in supportive care and other cancer services during first-line therapy that were not captured in the immunochemotherapy category. After first-line therapy, the cumulative incremental cost of other cancer care in the rituximab group declined, and by year three it was no longer statistically significant. This suggests there were some cost offsets possibly associated with lower rates of relapse in the rituximab group. There were no differences in the costs of noncancer services at any time during the observation period, suggesting that improved survival in the rituximab group had no appreciable impact on the costs of managing other diseases. Those with more advanced stage cancer and higher comorbidity burden also had higher costs, which is consistent with a priori expectations that increased patient complexity requires a higher level of care. The cost findings also suggest that the very elderly receive less first-line and subsequent immunochemotherapy.

The cumulative survival benefit of rituximab continued to accrue, and was statistically significant, throughout the observation period, and since the cumulative cost impact was negligible after the end of first-line therapy, the cost per life year gained declined (became more favorable for rituximab) rapidly during the observation period. Although we did not attempt to extrapolate our findings to a longer time period, it is likely that following these patients for a longer period would result in a lower cost-effectiveness ratio. Also, as we noted above, our study inclusion criteria required patients to have survived at least 60 days after the first claim for immunochemotherapy. Consequently, we did not account in our survival and cost-effectiveness analyses for patients who died during the first two months after starting first-line therapy. Neither pivotal phase III trial of first-line rituximab showed significant separation in the overall survival curves within the first several months after beginning therapy $[9,11]$. However, it is possible we would have observed larger survival differences, and hence smaller cost effectiveness ratios, had we included patients who died within the first two months of therapy. 


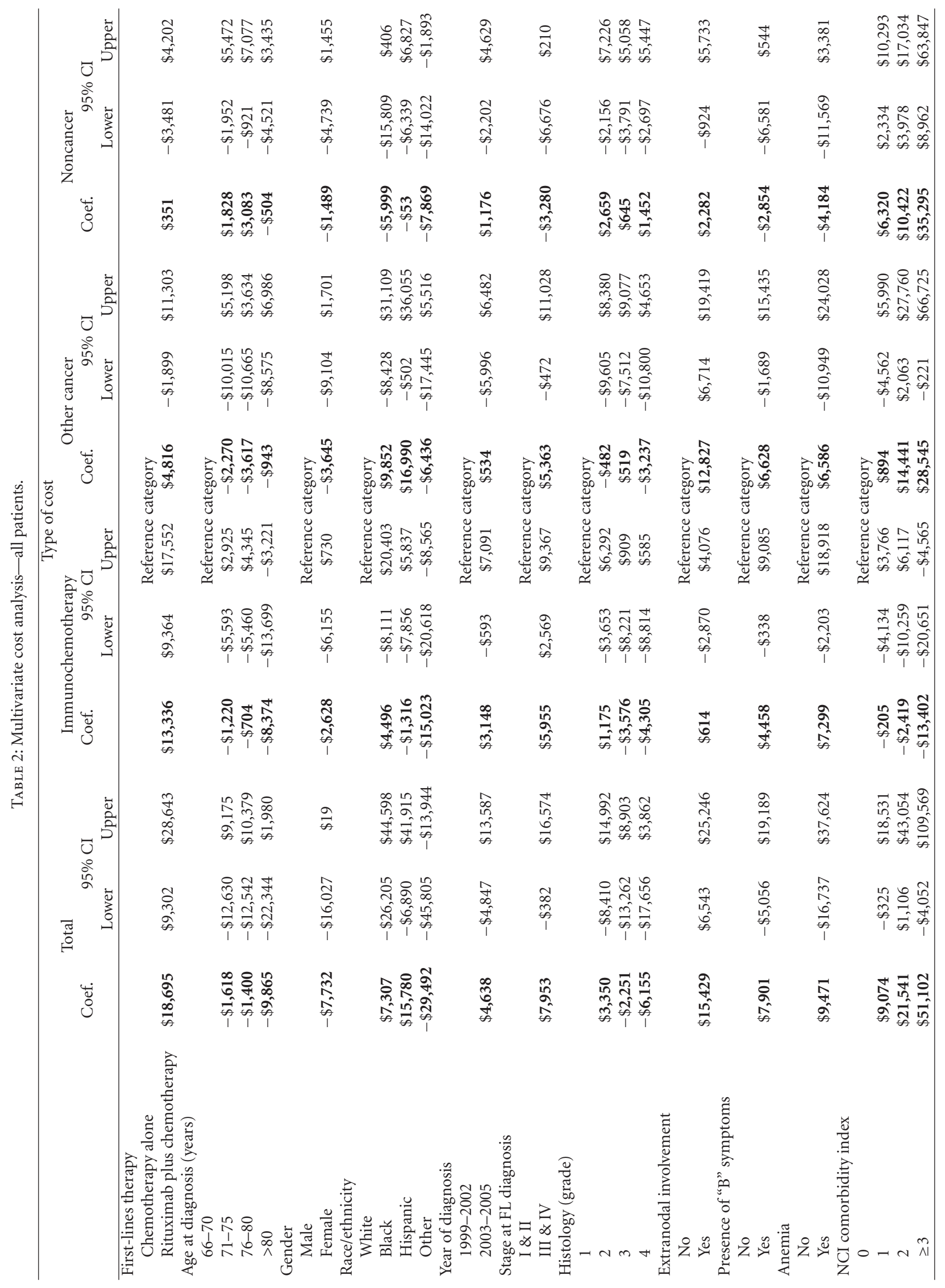




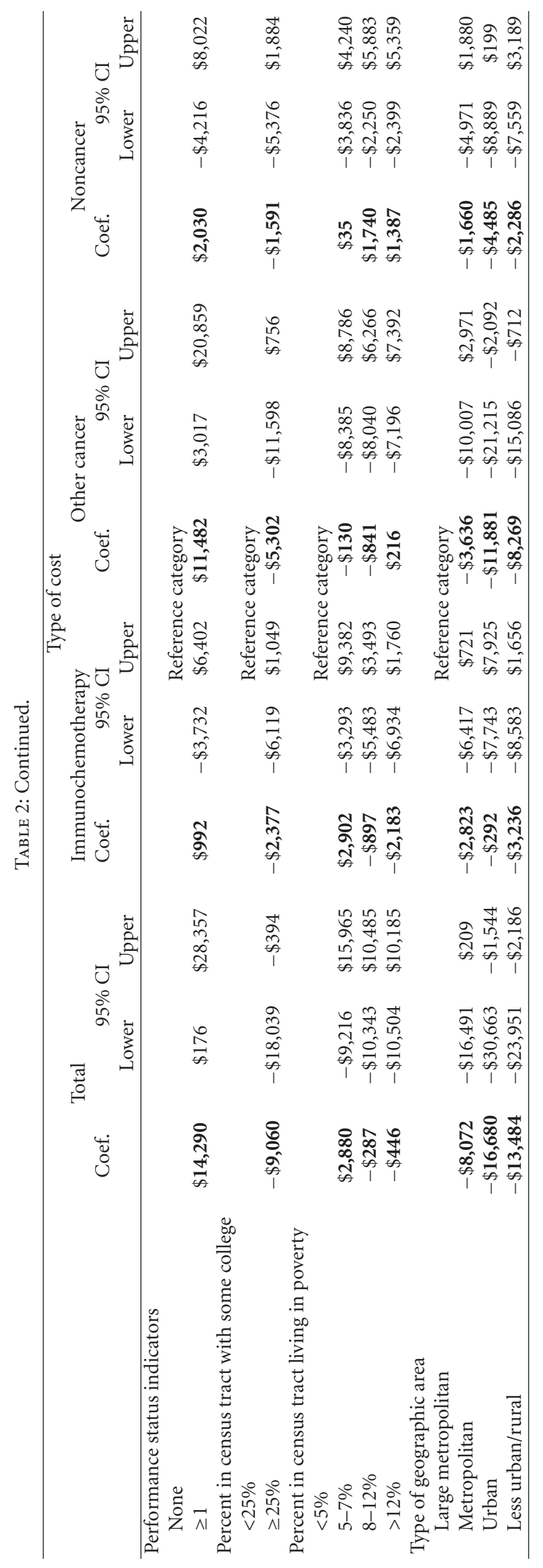


TABLE 3: Multivariate analysis-CHOP patients only.

\begin{tabular}{|c|c|c|c|}
\hline & & Total Cost & \\
\hline & Coef. & & \\
\hline & & Lower & Upper \\
\hline First-line therapy & & & \\
\hline Chemotherapy alone & & & \\
\hline Rituximab plus chemotherapy & $\$ 20,971$ & $\$ 9,089$ & $\$ 32,659$ \\
\hline Age at diagnosis (years) & & & \\
\hline $66-70$ & & & \\
\hline $71-75$ & $-\$ 2,912$ & $-\$ 15,466$ & $\$ 8,729$ \\
\hline $76-80$ & $\$ 773$ & $-\$ 11,864$ & $\$ 13,465$ \\
\hline$>80$ & $-\$ 7,929$ & $-\$ 22,218$ & $\$ 7,109$ \\
\hline Gender & & & \\
\hline Male & & & \\
\hline Female & $-\$ 3,782$ & $-\$ 13,154$ & $\$ 5,274$ \\
\hline Race/ethnicity & & & \\
\hline White & & & \\
\hline Black & $\$ 8,251$ & $-\$ 31,012$ & $\$ 52,301$ \\
\hline Hispanic & $\$ 21,353$ & $-\$ 9,712$ & $\$ 55,955$ \\
\hline Other & $-\$ 25,380$ & $-\$ 45,449$ & $-\$ 3,296$ \\
\hline Year of diagnosis & & & \\
\hline 1999-2002 & & & \\
\hline 2003-2005 & $-\$ 1,968$ & $-\$ 6,478$ & $\$ 2,424$ \\
\hline Stage at FL diagnosis & & & \\
\hline I \& II & & & \\
\hline III \& IV & $\$ 13,947$ & $\$ 3,943$ & $\$ 23,806$ \\
\hline Histology (grade) & & & \\
\hline 1 & & & \\
\hline 2 & $\$ 1,440$ & $-\$ 14,298$ & $\$ 18,830$ \\
\hline 3 & $-\$ 4,210$ & $-\$ 18,063$ & $\$ 10,882$ \\
\hline 4 & $-\$ 6,924$ & $-\$ 20,108$ & $\$ 6,774$ \\
\hline Extranodal involvement & & & \\
\hline No & & & \\
\hline Yes & $\$ 16,683$ & $\$ 6,307$ & $\$ 27,617$ \\
\hline Presence of " $B$ " symptoms & & & \\
\hline No & & & \\
\hline Yes & $\$ 7,654$ & $-\$ 6,707$ & $\$ 21,571$ \\
\hline Anemia & & & \\
\hline No & & & \\
\hline Yes & $\$ 24,622$ & $-\$ 7,946$ & $\$ 62,434$ \\
\hline NCI Comorbidity index & & & \\
\hline 0 & & & \\
\hline 1 & $\$ 7,999$ & $-\$ 3,871$ & $\$ 19,294$ \\
\hline 2 & $\$ 7,787$ & $-\$ 8,899$ & $\$ 26,799$ \\
\hline$\geq 3$ & $\$ 60,399$ & $-\$ 13,310$ & $\$ 137,762$ \\
\hline Performance status indicators & & & \\
\hline None & & & \\
\hline$\geq 1$ & $\$ 10,882$ & $-\$ 5,310$ & $\$ 26,939$ \\
\hline Percent in census tract with some & & & \\
\hline$<25 \%$ & & & \\
\hline$\geq 25 \%$ & $-\$ 4,875$ & $-\$ 15,504$ & $\$ 5,380$ \\
\hline
\end{tabular}


5

TABLE 3: Continued.

\begin{tabular}{lccr}
\hline & Coef. & Total Cost & \\
& & Lower & Upper \\
\hline $\begin{array}{l}\text { Percent in census tract living in poverty } \\
<5 \%\end{array}$ & & & \\
$5-7 \%$ & $\$ 2,021$ & $-\$ 12,003$ & $\$ 17,365$ \\
$8-12 \%$ & $\$ 5,296$ & $-\$ 7,066$ & $\$ 17,032$ \\
$>12 \%$ & $\$ 7,107$ & $-\$ 5,484$ & $\$ 18,882$ \\
Type of geographic area & & & \\
$\quad$ Large metropolitan & & & \\
$\quad$ Metropolitan & $-\$ 6,202$ & $-\$ 16,529$ & $\$ 4,807$ \\
$\quad$ Urban & $-\$ 14,949$ & $-\$ 33,514$ & $\$ 3,791$ \\
$\quad$ Less urban/rural & $-\$ 13,777$ & $-\$ 27,682$ & $\$ 49$ \\
\hline
\end{tabular}

SEER-Medicare does not include information on patient quality of life (QOL) or utility. Therefore, we did not adjust the survival estimates for QOL prior to calculating the costeffectiveness ratios. In their cost-effectiveness analysis based on a simulation model, Hornberger and colleagues [16] projected that adding rituximab to CVP would increase mean overall survival by 1.51 years and quality-adjusted lifeyears by 0.93 years, which suggests that had we adjusted our survival estimates for QOL, the cost-effectiveness ratios may have been larger than those based on unadjusted survival.

The main threat to the validity of our findings is selection bias, in which unobserved factors influence both treatment selection and the outcome of interest. For example, SEERMedicare does not contain data on hemoglobin level, LDH level, or the number of nodal areas, three of the five prognostic factors for mortality in the FLIPI [36]. We did, however, include age (all patients were older than the age threshold for excess risk of mortality in FLIPI $[\geq 60]$ ), stage (III-IV is associated with increased risk in FLIPI), and whether the patient had extranodal involvement. Also, we included a claims-based indicator of poor performance status [37]. In the multivariate analysis, $\geq 1$ indicator of poor performance, extranodal involvement, and later stage diagnosis all were associated with higher total and immunochemotherapy cost. It is important to note that any variable constructed using claims for medical services, such as performance status and NCI Comorbidity Index $[34,35]$, that is then included in a multivariate cost analysis, has a greater likelihood of being statistically significant simply by virtue of the fact that most claims have a cost attached.

In this study, patients diagnosed in later years were more likely to receive rituximab. This may have introduced several possible sources of bias including differential observation and censoring, and secular trends in both the assignment of patients to different chemotherapy regimens and in the use of supportive care. In the survival and cost analyses, we limited the observation period to reduce differential censoring between the treatment groups. We used IPTW in the estimation of cumulative incremental survival [46]. In the cost analysis, we used IPW and established separate weights for the two treatment groups to account for differential censoring between the two groups. Also, we included year of diagnosis as a covariable in the survival analysis and all the cost models to adjust for temporal differences in patterns of care. Most patients in both first-line treatment groups received CHOP chemotherapy. Therefore, we did not include type of chemotherapy in the primary analyses. When we restricted the cohort to those who received $\mathrm{CHOP}$, the cost coefficients for the rituximab group were similar to those in the primary analyses.

Our cost analyses were conducted from the perspective of Medicare. Therefore, we used Medicare paid amounts reported in claims to estimate total direct medical costs to Medicare. However, it is important to note that Medicare pays only $80 \%$ of part B outpatient costs. Therefore, total direct medical costs to all payers-including patients and other insurers-could be up to $20 \%$ higher than reported in this study. Also, Medicare did not pay for most oral drugs for the majority of the period in which our study was conducted, which is another reason why the costs reported in this study may underestimate the total direct medical costs of care to all payers. Also, it is likely that use of rituximab as maintenance therapy has increased in the years since the period covered by our data. It is unclear how this change in practice would affect our findings. Finally, the confidence intervals for the cost-effectiveness ratio were calculated by dividing the upper bound of the $95 \%$ CI for cost by the lower bound of the $95 \%$ CI for survival, and then repeating the process with the lower-cost and upper-survival bounds. In theory, bootstrapping the entire ratio is a preferred approach. However, to our knowledge such an approach has not been developed and reported in the literature.

\section{Conclusions}

In sum, adding rituximab to first-line chemotherapy for elderly patients with FL results in higher direct medical costs to Medicare and longer overall survival after four years. The net cost impact is due primarily to the treatment cost of first-line rituximab, although there may be small cost 
offsets associated with lower progression and/or relapse. The cost-effectiveness ratio we estimated is substantially higher than projected in a previous analysis based on a model. Extending the time horizon for our study, and including patients who died during the first two months of therapy may have resulted in a lower ratio. Our findings suggest even highly effective cancer therapies will do little to offset the rising costs of cancer care in the United States, and that additional public policies will be required to address this problem.

\section{Acknowledgments}

This study used the linked surveillance, epidemiology, and end results- (SEER-) Medicare database. The interpretation and reporting of these data are the sole responsibility of the authors. The authors acknowledge the efforts of the Applied Research Program, National Cancer Institute (NCI); the Office of Research, Development and Information, Centers for Medicare and Medicaid Services (CMS); Information Management Services (IMS), Inc.; the SEER Program tumor registries in the creation of the SEER-Medicare database. The authors would like to acknowledge the editorial assistance of Kim Merjan and Suzanne Griffiths in preparing this manuscript.

\section{References}

[1] B. E. Hillner and T. J. Smith, "Efficacy does not necessarily translate to cost effectiveness: a case study in the challenges associated with 21st-century cancer drug pricing," Journal of Clinical Oncology, vol. 27, no. 13, pp. 2111-2113, 2009.

[2] P. B. Bach, "Limits on medicare's ability to control rising spending on cancer drugs," The New England Journal of Medicine, vol. 360, no. 6, pp. 562-633, 2009.

[3] T. J. Smith and B. E. Hillner, "Bending the cost curve in cancer care," The New England Journal of Medicine, vol. 364, no. 21, pp. 2060-2065, 2011.

[4] Rituxan Timeline, http://www.gene.com/gene/products/information/oncology/rituxan/timeline.html.

[5] B. Coiffier, E. Lepage, J. Brière et al., "Chop chemotherapy plus rituximab compared with chop alone in elderly patients with diffuse large-B-cell lymphoma," The New England Journal of Medicine, vol. 346, no. 4, pp. 235-242, 2002.

[6] T. M. Habermann, E. A. Weller, V. A. Morrison et al., "Rituximab-CHOP versus $\mathrm{CHOP}$ alone or with maintenance rituximab in older patients with diffuse large B-cell lymphoma," Journal of Clinical Oncology, vol. 24, no. 19, pp. 3121-3127, 2006.

[7] M. Pfreundschuh, L. Trümper, A. Österborg et al., "CHOP-like chemotherapy plus rituximab versus CHOP-like chemotherapy alone in young patients with good-prognosis diffuse large-B-cell lymphoma: a randomised controlled trial by the MabThera International Trial (MInT) Group," The Lancet Oncology, vol. 7, no. 5, pp. 379-391, 2006.

[8] B. Coiffier, C. Thieblemont, E. Van Den Neste et al., "Longterm outcome of patients in the LNH-98.5 trial, the first randomized study comparing rituximab-CHOP to standard CHOP chemotherapy in DLBCL patients: a study by the Groupe d'Etudes des Lymphomes de l'Adulte," Blood, vol. 116, no. 12, pp. 2040-2045, 2010.
[9] W. Hiddemann, M. Kneba, M. Dreyling et al., "Frontline therapy with rituximab added to the combination of cyclophosphamide, doxorubicin, vincristine, and prednisone (CHOP) significantly improves the outcome for patients with advanced-stage follicular lymphoma compared with therapy with CHOP alone: results of a prospective randomized study of the German Low-Grade Lymphoma study group," Blood, vol. 106, no. 12, pp. 3725-3732, 2005.

[10] M. Herold, A. Haas, S. Srock et al., "Rituximab added to first-line mitoxantrone, chlorambucil, and prednisolone chemotherapy followed by interferon maintenance prolongs survival in patients with advanced follicular lymphoma: an East German study group hematology and oncology study," Journal of Clinical Oncology, vol. 25, no. 15, pp. 1986-1992, 2007.

[11] R. Marcus, K. Imrie, P. Solal-Celigny et al., "Phase III study of R-CVP compared with cyclophosphamide, vincristine, and prednisone alone in patients with previously untreated advanced follicular lymphoma," Journal of Clinical Oncology, vol. 26, no. 28, pp. 4579-4586, 2008.

[12] G. Salles, N. Mounier, S. de Guibert et al., "Rituximab combined with chemotherapy and interferon in follicular lymphoma patients: results of the GELA-GOELAMS FL2000 study," Blood, vol. 112, no. 13, pp. 4824-4831, 2008.

[13] R. Griffiths, M. Gleeson, C. Reyes, K. Knopf, and M. Danese, "Survival in elderly follicular lymphoma patients who receive frontline chemo-immunotherapy," American Journal of Hematology, vol. 85, no. 12, pp. 963-967, 2010.

[14] M. D. Danese, R. I. Griffiths, M. Gleeson et al., "An observational study of outcomes after initial infused therapy in medicare patients diagnosed with chronic lymphocytic leukemia," Blood, vol. 117, no. 13, pp. 3505-3513, 2011.

[15] R. Griffiths, J. Mikhael, M. Gleeson, M. Danese, and M. Dreyling, "Addition of rituximab to chemotherapy alone as first-line therapy improves overall survival in elderly patients with mantle cell lymphoma," Blood, vol. 118, no. 18, pp. 48084816, 2011.

[16] J. Hornberger, C. Reyes, D. Lubeck, and N. Valente, "Economic evaluation of rituximab plus cyclophosphamide, vincristine and prednisolone for advanced follicular lymphoma," Leukemia and Lymphoma, vol. 49, no. 2, pp. 227-236, 2008.

[17] J. C. Hornberger and J. H. Best, "Cost utility in the United States of rituximab plus cyclophosphamide, doxorubicin, vincristine, and prednisone for the treatment of elderly patients with diffuse large B-cell lymphoma," Cancer, vol. 103, no. 8, pp. 1644-1651, 2005.

[18] U. S. Department of Health, Human Services Centers for Medicare, and Medicaid Services, Medicare Part B Drug Average Sales Price. 2011 ASP Drug Pricing Files, https://www. cms.gov/McrPartBDrugAvgSalesPrice/01a18_2011aspfiles.asp, 2011.

[19] Rituximab package insert, http://www.gene.com/gene/products/information/pdf/rituxan-prescribing.pdf.

[20] National Comprehensive Cancer Network, NCCN Clinical Practice Guidelines in Oncology Non-Hodgkin's Lymphomas V.1.2010.

[21] Surveillance, Epidemiology, and End Results (SEER) Program, http://www.seer.cancer.gov/, SEER*Stat Database: Incidence-SEER 17 Regs Research Data + Hurricane Katrina Impacted Louisiana Cases, Nov 2010 Sub (2000-2008)Linked To County Attributes-Total U.S., 1969-2009 Counties, National Cancer Institute, DCCPS, Surveillance Research Program, Cancer Statistics Branch, released April 2011, based on the November 2010 submission. 
[22] Surveillance, Epidemiology, and End Results (SEER) Program, http://www.seer.cancer.gov/, SEER*Stat Database: Incidence-SEER 9 Regs Research Data, Nov 2010 Sub (1973-2008)¡Katrina/Rita Population Adjustment>-Linked To County Attributes-Total U.S., 1969-2009 Counties, National Cancer Institute, DCCPS, Surveillance Research Program, Cancer Statistics Branch, released April 2011, based on the November 2010 submission.

[23] Medicare Payment Advisory Commission (Medpac), June 2011, A Data Book: Health Care Spending and the Medicare Program, http://www.medpac.gov/chapters/Jun11DataBookSec10.pdf.

[24] M. T. Groot, P. J. Lugtenburg, J. Hornberger, P. C. Huijgens, and C. A. Uyl-De Groot, "Cost-effectiveness of rituximab (MabThera) in diffuse large B-cell lymphoma in the Netherlands," European Journal of Haematology, vol. 74, no. 3, pp. 194-202, 2005.

[25] J. H. Best, J. Hornberger, S. J. Proctor, L. F. Omnes, and F. Jost, "Cost-effectiveness analysis of rituximab combined with CHOP for treatment of diffuse large B-cell lymphoma," Value in Health, vol. 8, no. 4, pp. 462-470, 2005.

[26] F. Ferrara and R. Ravasio, "Cost-effectiveness analysis of the addition of rituximab to CHOP in young patients with good-prognosis diffuse large-B-cell lymphoma," Clinical Drug Investigation, vol. 28, no. 1, pp. 55-65, 2008.

[27] K. M. Johnston, C. A. Marra, J. M. Connors, M. Najafzadeh, L. Sehn, and S. J. Peacock, "Cost-effectiveness of the addition of rituximab to $\mathrm{CHOP}$ chemotherapy in first-line treatment for diffuse large B-cell lymphoma in a population-based observational cohort in British Columbia, Canada," Value in Health, vol. 13, no. 6, pp. 703-711, 2010.

[28] J. A. Ray, E. Carr, G. Lewis, and R. Marcus, "An evaluation of the cost-effectiveness of rituximab in combination with chemotherapy for the first-line treatment of follicular nonHodgkin's lymphoma in the UK," Value in Health, vol. 13, no. 4, pp. 346-357, 2010.

[29] R. I. Griffiths, M. L. Gleeson, J. R. Mikhael, and M. D. Danese, "Long-term direct medical costs in patients diagnosed with follicular lymphoma who receive frontline chemotherapy with versus without rituximab-a SEER-medicare analysis," in Proceedings of the ISPOR 16th Annual International Meeting, Baltimore, Md, USA, May 2011.

[30] J. L. Warren, C. N. Klabunde, D. Schrag, P. B. Bach, and G. F. Riley, "Overview of the SEER-medicare data: content, research applications, and generalizability to the United States elderly population," Medical Care, vol. 40, no. 8, pp. IV-3-IV-18, 2002.

[31] National Cancer Institute, Overview of the SEER Program, Bethesda, Md, USA, National Cancer Institute, http://seer .cancer.gov/about/overview.html.

[32] National Cancer Institute, Overview of the SEER Program, Bethesda, Md, USA: National Cancer Institute, http://healthservices.cancer.gov/seermedicare/overview/linked.html, 2011.

[33] North American Association of Central Cancer Registries, Guidelines for ICD-O-3 Implementation, 2000.

[34] C. N. Klabunde, A. L. Potosky, J. M. Legler, and J. L. Warren, "Development of a comorbidity index using physician claims data," Journal of Clinical Epidemiology, vol. 53, no. 12, pp. 1258-1267, 2000.

[35] C. N. Klabunde, J. M. Legler, J. L. Warren, L. M. Baldwin, and D. Schrag, "A refined comorbidity measurement algorithm for claims-based studies of breast, prostate, colorectal, and lung cancer patients," Annals of Epidemiology, vol. 17, no. 8, pp. 584-590, 2007.
[36] P. Solal-Céligny, P. Roy, P. Colombat et al., "Follicular lymphoma international prognostic index," Blood, vol. 104, no. 5, pp. 1258-1265, 2004.

[37] A. J. Davidoff, M. Tang, B. Seal, and M. J. Edelman, "Chemotherapy and survival benefit in elderly patients with advanced non-small-cell lung cancer," Journal of Clinical Oncology, vol. 28, no. 13, pp. 2191-2197, 2010.

[38] K. R. Yabroff, E. B. Lamont, A. Mariotto et al., "Cost of care for elderly cancer patients in the United States," Journal of the National Cancer Institute, vol. 100, no. 9, pp. 630-641, 2008.

[39] D. Y. Lin, "Linear regression analysis of censored medical costs," Biostatistics, vol. 1, pp. 35-47, 2000.

[40] H. Bang and A. A. Tsiatis, "Estimating medical costs with censored data," Biometrika, vol. 87, no. 2, pp. 329-343, 2000.

[41] A. O'Hagan and J. W. Stevens, "On estimators of medical costs with censored data," Journal of Health Economics, vol. 23, no. 3, pp. 615-625, 2004.

[42] M. Raikou and A. McGuire, "Estimating medical care costs under conditions of censoring," Journal of Health Economics, vol. 23, no. 3, pp. 443-470, 2004.

[43] T. A. Young, "Estimating mean total costs in the presence of censoring: a comparative assessment of methods," PharmacoEconomics, vol. 23, no. 12, pp. 1229-1242, 2005.

[44] A. Basu and W. G. Manning, "Estimating lifetime or episodeof-illness costs under censoring," Health Economics, vol. 19, no. 9, pp. 1010-1028, 2010.

[45] R. I. Griffiths, R. L. Barron, M. L. Gleeson et al., "Granulocytecolony stimulating factor (G-CSF) use and medical costs after initial adjuvant chemotherapy in older patients with earlystage breast cancer (ESBC)," PharmacoEconomics, vol. 30, no. 2, pp. 103-118, 2011.

[46] J. Xie and C. Liu, "Adjusted Kaplan-Meier estimator and logrank test with inverse probability of treatment weighting for survival data," Statistics in Medicine, vol. 24, no. 20, pp. 30893110, 2005.

[47] B. Efron and R. J. Tibshirani, An Introduction to the Bootstrap (Monographs on Statistics and Applied Probability), Chapman and Hall/CRC, Boca Raton, Fla, USA, 1994. 


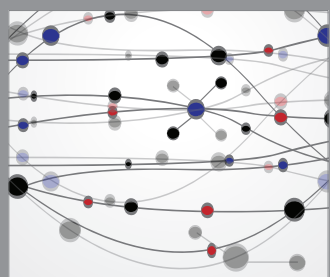

The Scientific World Journal
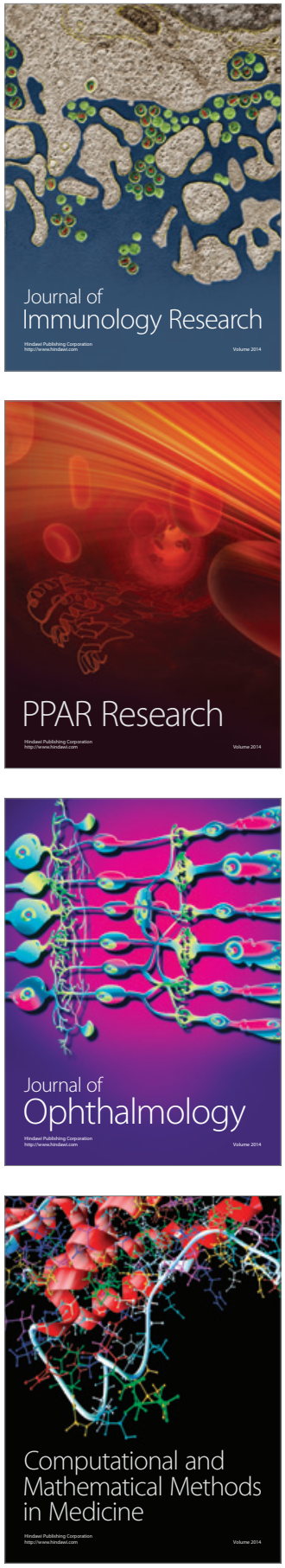

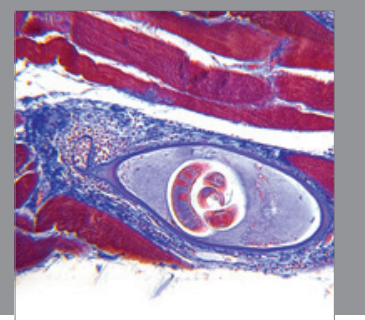

Gastroenterology

Research and Practice
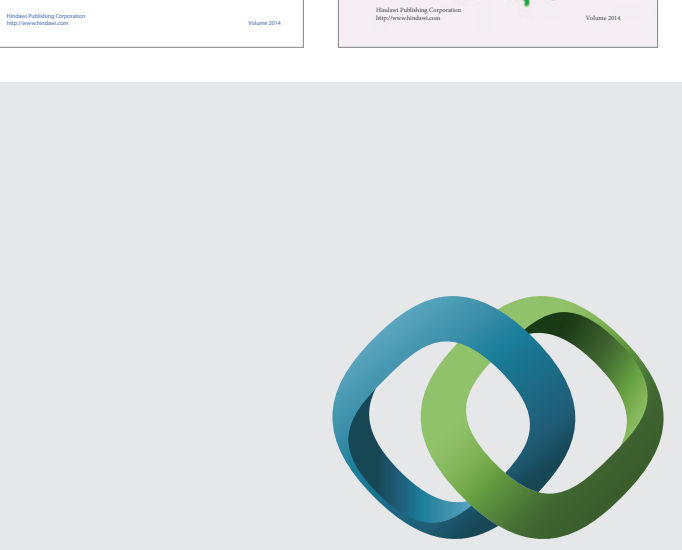

\section{Hindawi}

Submit your manuscripts at

http://www.hindawi.com
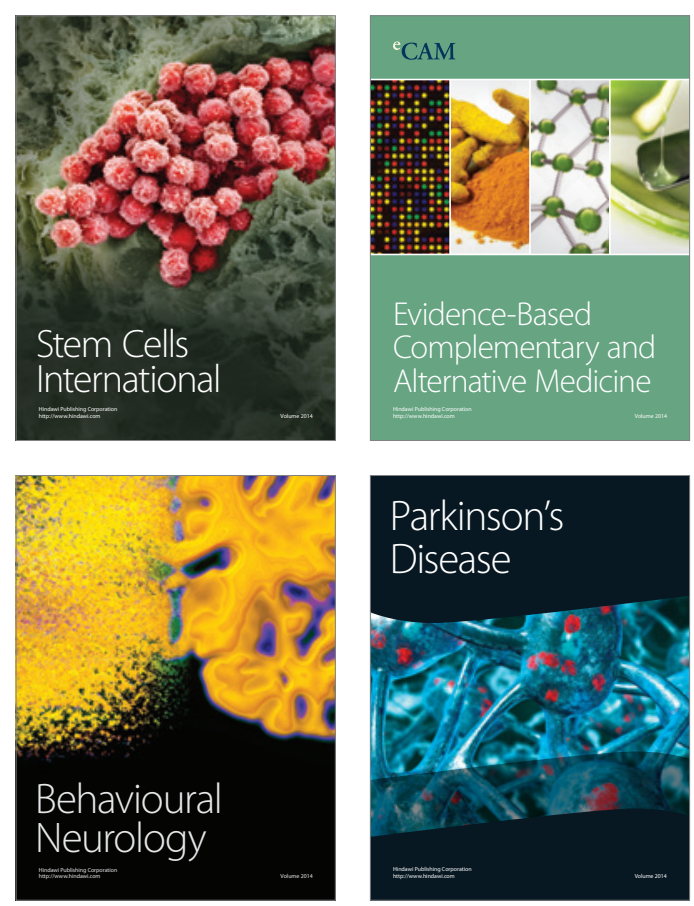

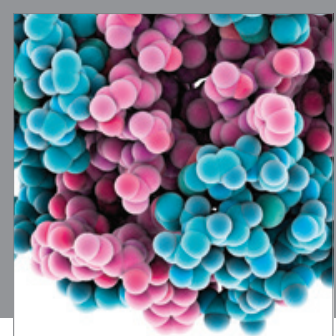

Journal of
Diabetes Research

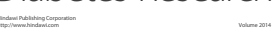

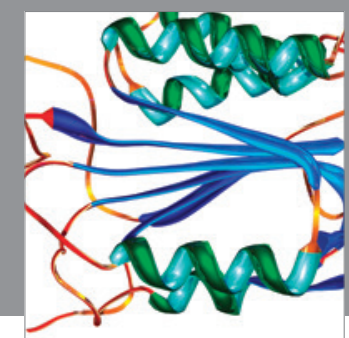

Disease Markers
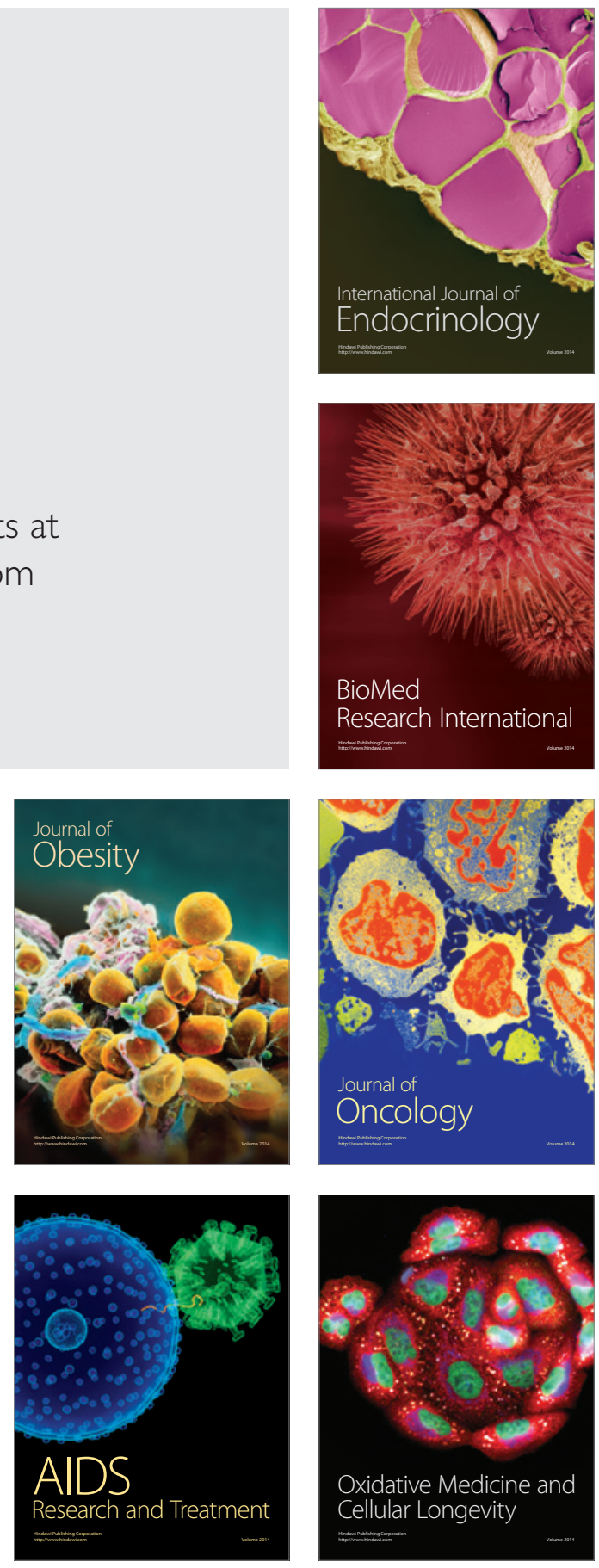